\title{
PMU Orchestrator as a solution for managing microgrid monitoring with $5 \mathrm{G}$ communication
}

\author{
Mihai Sanduleac \\ Centrul Român al Energiei \\ University Politehnica of Bucharest \\ Bucharest, Romania \\ mihai.sanduleac@gmail.com
}

\author{
Catalin Chimirel \\ Centrul Român al Energiei \\ Bucharest, Romania \\ catalin.chimirel@crenerg.org
}

\author{
Mihai Paun \\ Centrul Român al Energiei \\ Bucharest, Romania \\ mihai.paun@crenerg.org
}

\begin{abstract}
In high voltage networks Phasor Measurement Units (PMU) are already used for purposes such as assessing angle stability, detecting areas of high production versus areas of high consumption or for analysis during grid disturbances. However, there are less studies regarding the benefits of PMUs in low voltage networks. PMUs are promising devices for use in active distribution grid, to help monitoring and control in a scenario with high renewables penetration. The paper is analyzing possible benefits from using low-cost PMUs in low voltage microgrids, supported also by the upcoming high end $5 \mathrm{G}$ communication, to facilitate microgrid monitoring in real time while giving also useful information processed from PMU data.
\end{abstract}

Keywords-PMU, data orchestration, active distribution network, microgrid, NORM, unbundled smart meter

\section{INTRODUCTION}

Phasor Measurement Units (PMUs) are promising devices for use in active distribution grid, to help monitoring and control in scenarios with high renewables penetration.

Their potential of being used in the distribution grid has been already analysed in several papers. The necessity and benefits of using PMUs in distribution grids is analysed in [1]. In [2] it is reported a so called Angle Constraint Active Management, where a renewable generation is constrained based on voltage angle difference signals produced by Phasor Measurement Units placed in a medium voltage distribution network.

PMUs used for fault location in active distribution network, based on the change of current phase, are studied through simulations in [3], where it is assumed that several PMUs are installed in a distribution network and phasors can be measured on lines. By using the PMU data, the paper shows that the change of the current phase difference of a specific line has the potential of being a fault location criterion, as a differentiation between normal operation and fault conditions. Similar subject is treated in [4], where faults can be identified within $1 \%$ accuracy of the line length.

Using PMU data for improving state estimation accuracy is another domain where active distribution networks, e.g. with high PV penetration, can be better monitored and controlled [5]. The role of appropriate PMU placement in order to improve Distribution System State Estimation is analysed in [6].

A general view targeting data integration is approached in [7], where the analysis is focusing on the use of new smart meter design and of new phasor measurement unit (PMU) and power quality analysers, in order to perform necessary observability in active distribution networks. Moreover, using interoperability of power system protection by using SCADA and PMU is shown to bring advantages in order to achieve EMS-SCADA functionalities [8]. Integration between SCADA and PMU data is also presented in other papers, such as [9], using an open source SCADA implementation.

The rich content of PMUs is also a subject of new aggregation algorithms to be used in active distribution grids for aggregating frequency, rate of change of frequency and unbalance coefficient [10].

Data inconsistency based on PMU information is another aspect of consolidating active distribution grid observability and aspects related to this potential are presented in other works.

New options for voltage-to-power sensitivity estimation and voltage regulation are studied in [11], where PMUs are considered for an augmented sensitivity estimation-based voltage control strategy, with case studies and analysis on the IEEE 33-node test feeder.

PMUs are traditionally expensive equipment and finding appropriate communication infrastructure to send data to a Phasor Data Concentrator (PDC) is also a challenging task, from both bandwidth and availability points of view. For this reason, there are still implementations which consider mobile fleet of PMUs to be placed in different places of the network for a certain period of time, in order to analyse specific situations [12].

Active distribution networks are the emerging domain for using PMU data in many applications, some of them presented above. However, price of the equipment, communication affordability and service-oriented requests are different challenging aspects which still need to be addressed. There are needed different enabling technologies to be brought on market, in order to unleash the PMU potential. Between these enablers, we can consider:

a) a low-cost solution to integrate the smart meter needs but also the necessity for phasors measurement; incorporating a smart meter, a low-cost PMU and an energy gateway to connect to external environment can be a solution for having one integrated equipment placed on the point of common coupling (PCC) of each consumer or prosumer; based on this approach is designed the NORM equipment developed in Success project [13];

b) the $5 \mathrm{G}$ technology, allowing high band and low latency, thus allowing real-time receival of PMU data e.g. each second and the possibility to make aggregated assessment on all the data received; 
c) $5 \mathrm{G}$ oriented virtual services acting as "Smart Energyas-a-Service", which bring the necessary security and pluggability needed for applications with many participating devices.

NORM is based on an unbundled smart meter (USM) architecture, where metrology part is separated from the business part, allowing incremental functionality on the latest, including new $5 \mathrm{G}$ interfacing and $5 \mathrm{G}$ oriented virtual services. The 5G potential of NORM has been developed in [14]. Aspects related to data inconsistency assessment in a distribution grid, based on NORM architecture, has been presented in [15].

The paper presents an PMU orchestrator application which considers NORM equipment with integrated PMU mounted in all points of a low voltage distribution grid with high renewables resources (RES) penetration, as an application to monitor and assess through data aggregation the status of the active distribution network organised as a microgrid entity.

\section{MICROGRID ENVIRONMENT}

In order to study the PMU data aggregation, it is needed to have a microgrid with characteristics which can show specific dynamics of an active distribution grid. This means that during an entire day there are time-periods when the grid has normal power flow from the $\mathrm{MV} / \mathrm{LV}$ transformer to the endcustomers, and that in other time periods the power flow may see power injection back to the medium voltage grid.

In order to be able to perform appropriate analysis, it has been selected a low voltage microgrid used in University Politehnica of Bucharest (UPB) for laboratory studies.
Figure 1 shows the microgrid chosen for the analysis. Particularly it is made an analysis for the MG.1-LV grid, which is in default operation the whole network supplied by transformer T1, without connection to MG.2-LV, as usually the breaker $\mathrm{K} 2$ is open.

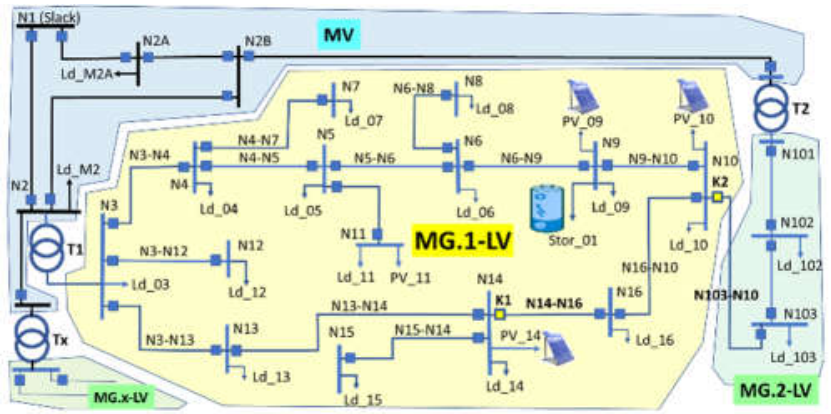

Figure 1 - Microgrid topology used in the paper analysis.

The analysis has been made by using the open source loadflow application OpenDSS [16][17] and the application environment for managing power grid studies named GridMonK [18], developed as basic functionalities by UPB and with a specific module developed for NRG5. The setup allowed to simulate the whole microgrid as providing PMU data in all grid points.

The simulated data environment considered for the PMU orchestrator is presented in Figure 2. The Figure shows the GridMonK interface with the microgrid, with most of the load-flow results displayed in the specific objects shown as coloured rectangles: lines (white rectangles), loads (yellow), PV production units (green), storage resources (half green, half yellow), electric vehicles (pink), transformers (light blue).

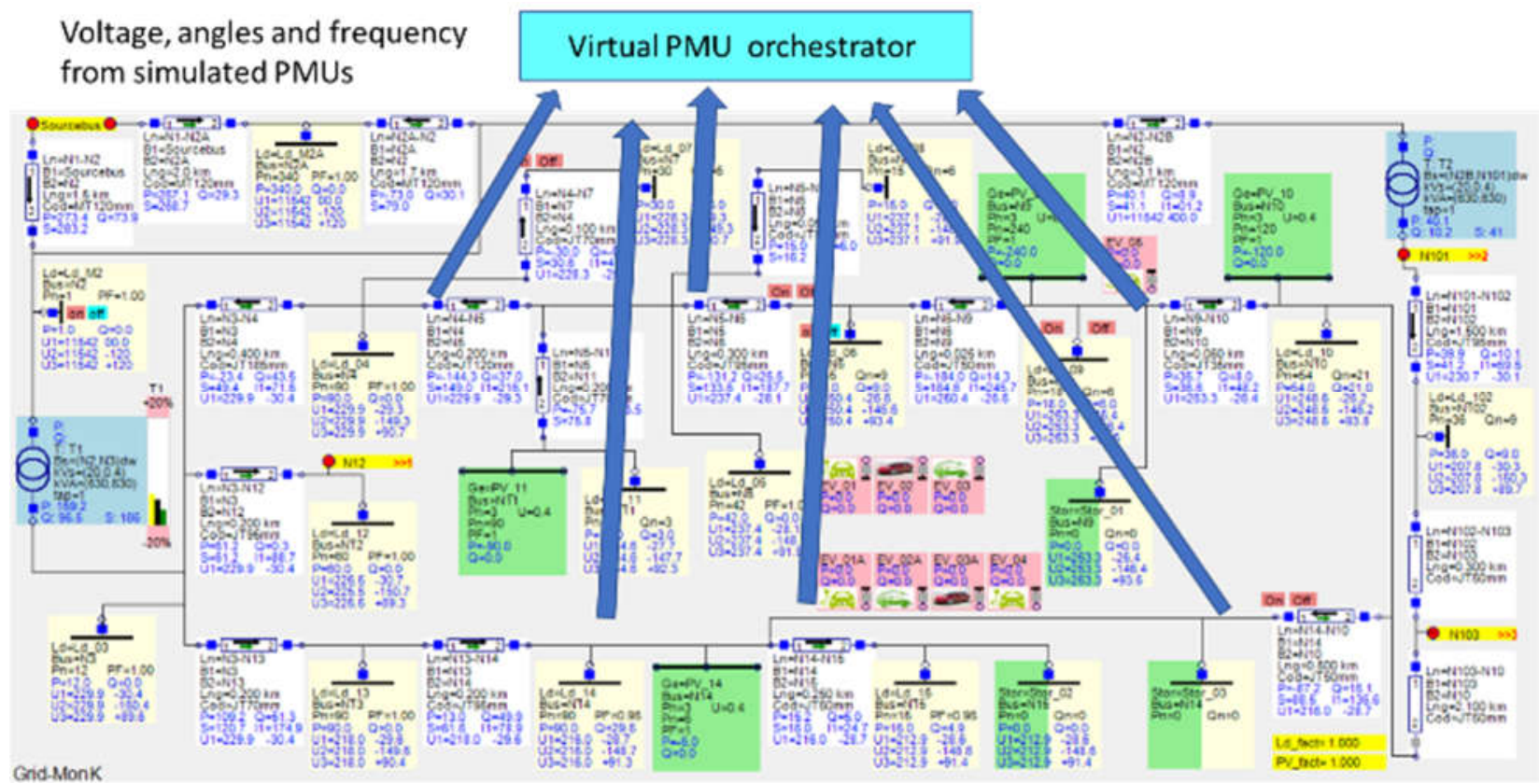

Figure 1 - Microgrid setup with calculated values and interaction with the vPMU Orchestrator

In this setup, the angles of voltages are considered to be measured by PMUs in all grid points, in a scenario with meters having in all places an advanced NORM architecture. For convenience, power flow direction is also depicted in the line drawings. The Figure show that the Virtual PMU (vPMU) orchestrator is the specific module of GridMonK which is acquiring the voltage phase data from all the metering points, as inputs for the microgrid monitoring and assessment. The data transmission is simulated inside the application (large blue arrows pointing to the upper part where vPMU orchestrator is depicted), however, it is considered to be a $5 \mathrm{G}$ 
communication to support the vPMU orchestrator in real deployments.

Angle values are measured having the Sourcebus as reference, acting also as Slack bus in the power flow calculation, thus with voltage zero angle. As the transformer $\mathrm{T} 1$ is near to the Sourcebus, it can be considered at microgrid level as being similar to a Slack bus, however, due to the characteristics of the MV/LV transformer, the angle on the LV side of the transformer has a value around -30 degrees, which is used in the microgrid as reference of our analysis. For particular cases, the observed angle values are in situations as presented in Figures 2 and 3. Based on their sign and value, the angles show change of load flow direction, allowing a characterisation of the microgrid as being in a consumption predominant (Figure 2) or production predominant situation (Figure 3).

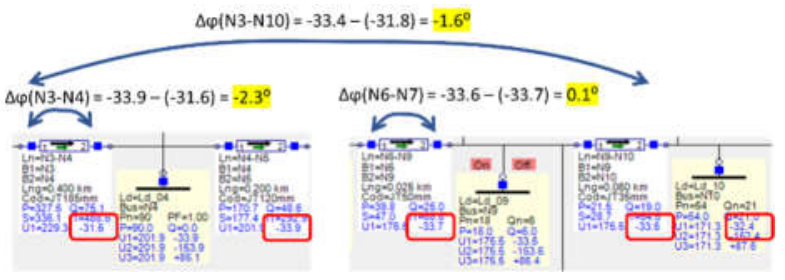

Figure 2 - Usual load-flow in a consumption predominant situation

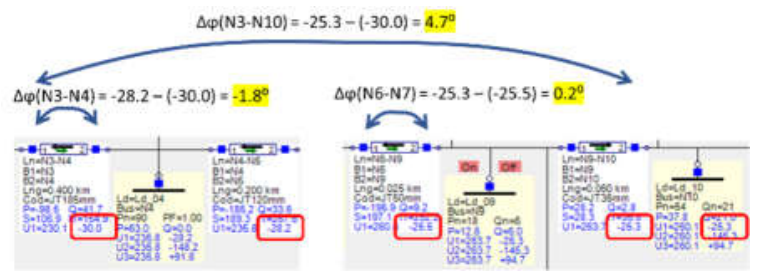

Figure 3 - Reversed load-flow in a high production situation

With this situation, the observability enabled by the PMU voltage angle data becomes similar with the one found in the transmission network, showing that the active distribution networks need to implement in an adapted way methods similar with the ones traditionally found at transmission level.

\section{SCENARIOS AND THEIR MODELATION} day:

The following scenarios are considered during the studied

- Basic scenario 1, with $\mathrm{K} 1$ and K2 open, meaning that the MG.1-LV grid is working in radial mode, without any connection with MG.2-LV grid.

- Scenario 2, with K1 closed K2 open, meaning that the MG.1-LV operates with a loop between the feeder starting with line N3-N4 and the feeder starting with the line N3-N13;in this scenario there is still no connection with MG.2-LV grid;

- Scenario 3, with K1 open and K2 closed, meaning that the MG.1-LV grid is working in radial mode, but there is a connection with MG.2-LV grid through K2.

- Scenario 4, with K1 and K2 closed, meaning that the MG.1-LV grid is working in LV loop mode, and there is a connection with MG.2-LV grid through $\mathrm{K} 2$.

During each scenario the most important periods are: a) midday period, having high distributed production and lower consumption; this is typical for the period [12:00h 15:00h];

b) evening peak, having high consumption and low to zero production; this is typical e.g. for the period [19:00h - 22:00h]

The scenarios are incorporated in a full day evolution for both consumption as well as production, based on one-minute records for both production and consumption. In this way it is analysed a near real-time situation, capturing quick evolutions of both production and consumption.

\section{PMU ORCHESTRATION ALGORITHMS}

As data from PMUs are considered to come at higher rate possible (from each 1 minute in our simulation, down to subsecond reporting time in real environment), the tests are simulating a similar set of PMU data received at each loadflow calculation.

The following stream of data is received from the simulated microgrid after each load-flow calculation:

$\operatorname{PMU}[\mathrm{t}]=\left\{[\mathrm{v} 1(\mathrm{t}), \varphi 1(\mathrm{t})],[\mathrm{v} 2(\mathrm{t}), \varphi 2(\mathrm{t})], . .\left[\mathrm{v}_{\mathrm{N}}(\mathrm{t}), \varphi_{\mathrm{N}}(\mathrm{t})\right]\right\}$

The PMU data set is then analysed by selecting special grid points or by using statistical means to describe a cluster of nodes. For the latest, it is first applied a clustering procedure, which is mainly based on the topology of the grid. Based on the selected nodes or on their equivalent in clusters, the following data are considered:

- node value in selected nodes or mean value of voltage angle in each cluster

- inter-node or inter-cluster angle difference

The voltage angle data is calculated each time period and the vPMU orchestrator is assessing the grid status after each calculation.

\section{SIMULATION RESULTS AND INTERPRETATION}

The load-flow has been calculated on a daily basis using 1440 different regime situations, meaning a regime for each one minute. However, the consumption and production profiles considered in the simulation constant values over an hour, thus results will show also constant values over each hourly interval. A set of PMU data has been generated for the entire period, in order to make possible an microgrid analysis based on PMU data. Figure 4 shows a simple interface of the vPMU orchestrator, displaying relevant data for the whole microgrid.

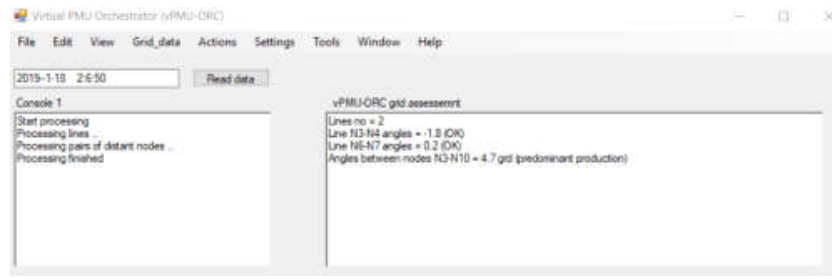

Figure 4 - Reporting console for the PMU orchestrator

In Figure 5 it is depicted one of the typical situations of an active distribution grid, showing reverse power flow in some of the grid lines nearby the transformer and through the 
transformer. The situation occurs in a midday peak with microgrid high production and lower consumption.

In order to give an image of the microgrid observability using PMU data, a full 24 hours analysis has been made.

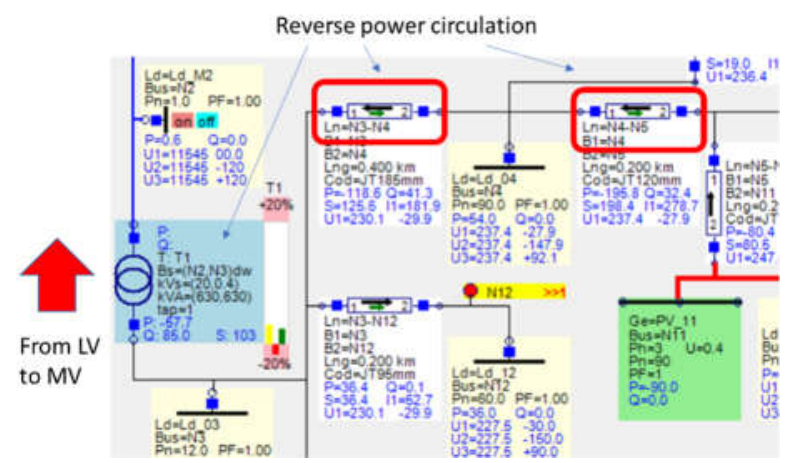

Figure 5 - Reverse power flow during excess of production

The evolution of voltage phase angles over the period at the distant node N10 (Figure 1) are presented in Figure 6, for each of the scenarios. It can be seen that the excess production and excess consumption can be easily deducted from the evolution of the voltage angle difference form the transformer LV connections. The evolution is similar for each of the four scenarios, showing a strong correlation between the angle sign and the excess production (positive sign) and excess consumption (negative sign).

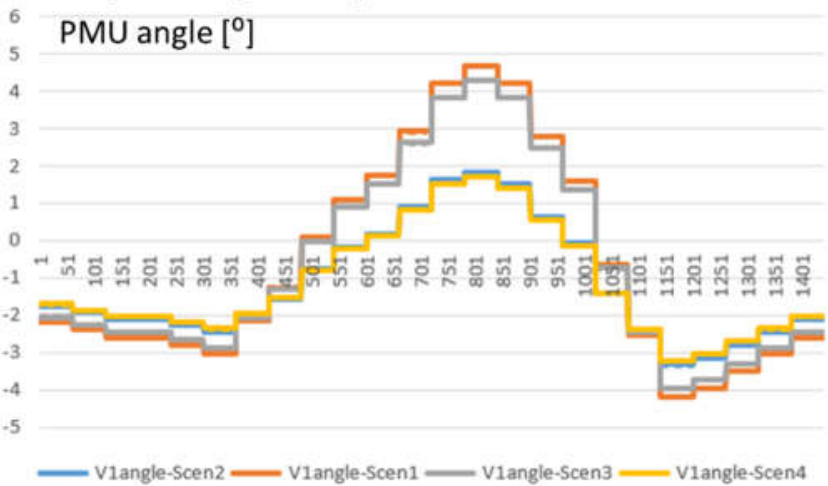

Figure 6 - PMU voltage phase evolution in four scenarios

Figure 7 presents a variation of the Scenario 1, comparing the basic situation (V1-Scen1) with a setup in which the battery in node N9 has a scheduled task, meaning it absorbs energy during midday period and release it during evening peak and remaining periods, to accomplish a complete battery cycle.

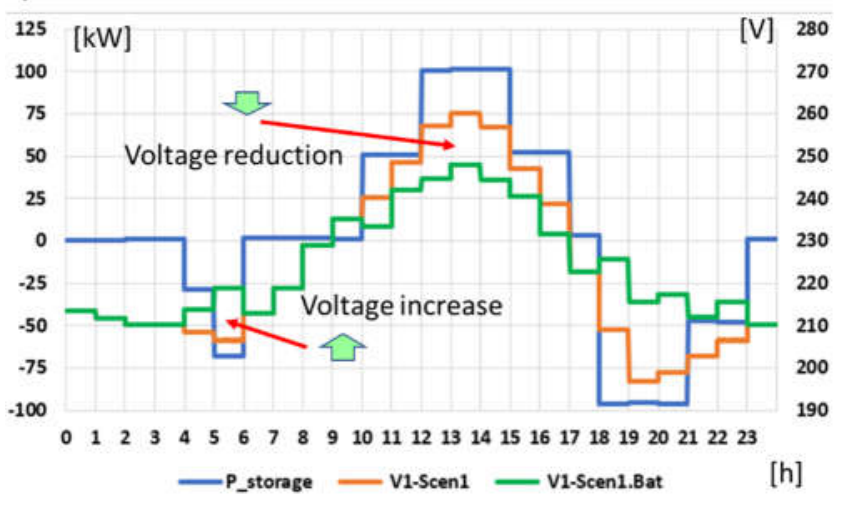

Figure 7 - Battery schedule and impact on voltage level
The voltage variation during time, for the battery scheduled for $\mathrm{P}$ _storage has the evolution presented with green line (V1-Scen1.Bat). It can be seen the benefic impact of the storage resource in the microgrid voltage level.

Figure 8 presents a comparison of the evolution of the voltage angle of node N10 in both Scenario 1 situations: with and without battery in N9. It can be seen that with activated battery schedule there a lower variation of voltage angle, however the shape remains similar with the basic situation, showing that there is still a change during the day in $\mathrm{N} 10$, between excess production and excess consumption.

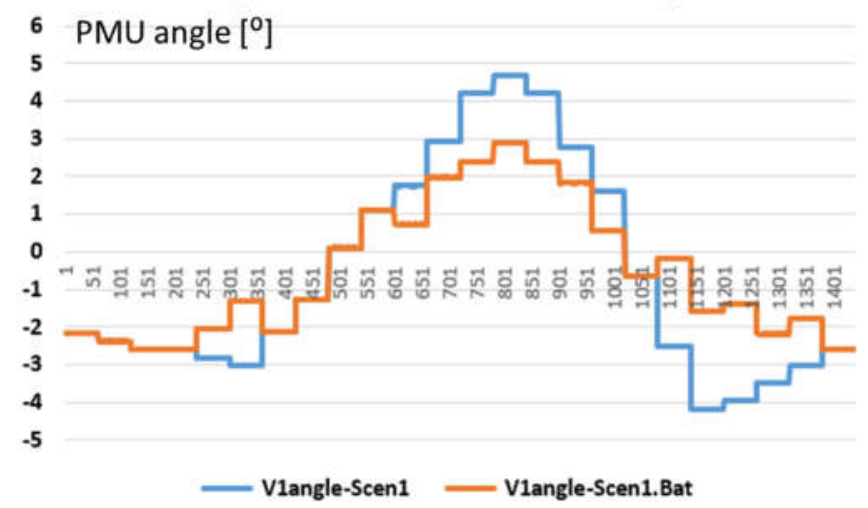

Figure 8 - PMU evolution of Scenario 1 without and with battery.

Figure 9 depicts the same evolution from Figure 7, but in a circular presentation, having the green circle as "boundary between excess consumption (into the circle) and production (outside the circle). This can be another view of microgrid monitoring using a PMU orchestrator. Such view may be more easily interpreted by a future microgrid operator.

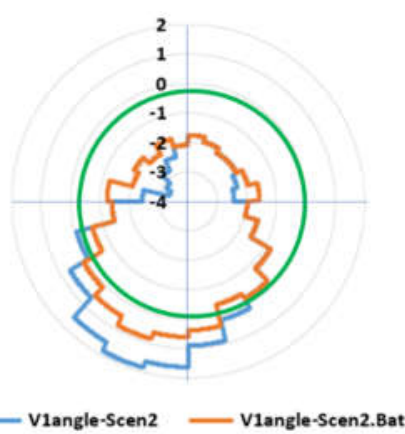

Figure 9 - PMU data evolution in a circular format.

Figure 10 shows similar comparisons between situations with or without battery schedule, in the Scenario 2 situation.

3 PMU angle $\left[{ }^{\circ}\right]$

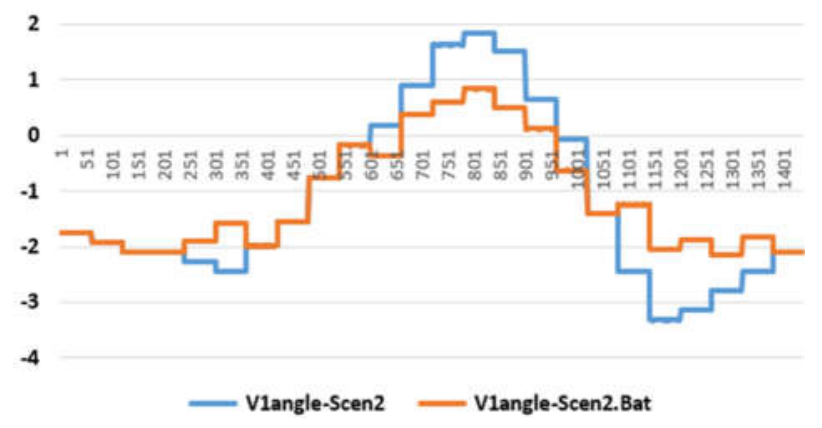

Figure $10-$ PMU evolution of Scenario 2 without and with battery. 
For the analyzed setup, in all scenarios it can be observed that the voltage angle evolution has only a small range of up to \pm 6 degrees. These results confirm the fact that in LV networks it is more difficult to measure PMU data, because the $\mathrm{R} / \mathrm{X}$ ratio is not in favor of having bigger voltage angles variations and the distance between nodes (length of the lines) is also smaller than in high voltage networks.

In low voltage microgrids PMUs need therefore to comply with harder accuracy requirements, while it is also expected to have lower prices for the measurements.

Promising results can be however obtained within our study: a remote node can "feel" the nature of the grid situation, meaning excess of produced energy or excess of consumption, by simply comparing the voltage angle of its node with the voltage angle of the grid former, which is in this case the low side of the transformer.

If the PMU at transformer side is sending its synchronized angle to all participants, in a broadcasting way, then it is no need in the remote node to have information from other participants, but to simply follow the voltage angle evolution. A $5 \mathrm{G}$ communication network is a good enabler for such functionality.

As this voltage angle difference is a sort of unbalance information seen from the local PMU, it may be possible to have balancing services based on this information.

\section{CONCLUSIONS AND FUTURE WORK}

The paper presents the low voltage microgrid PMU Orchestrator as a solution for managing microgrid monitoring, helped by $5 \mathrm{G}$ communication, to allow real-time assessment. The analysis has been made by simulation, using open source components OpenDSS and GridMonK.

It has been selected a specific microgrid and four different scenarios have been used over full days, at one-minute resolution. Variations with storage means in a s0pecific node have been also presented. Second or sub-second based dynamics can work in similar way, with the support of proper communication, such as $5 \mathrm{G}$ local infrastructure.

The results suggests a good dependency of voltage angles with the excess production / excess consumption status, in specific nodes, showing potential for microgrid observability support using PMU data.

As the subject of using PMU data aggregation is still a new topic for the low voltage microgrids, we see possible future work in different directions, such as:

- 5G communication impact on microgrid observability, impact of its small latency, of the high band; conditions to have resilient local support through the local $5 \mathrm{G}$ tower, with specific embedded intelligence.

- Enhance grid simulation to analyze PMU data in dynamic situations

- Use the setup for training purposes, in order to assess specific challenges of future microgrids with high penetration of RES; the environment needed to assess the PMU orchestrator can be not only a tool for analysis, but can become also a training toll for a so-called microgrid dispatch service.

\section{ACKNOWLEDGMENT}

The research work leading to these results has received funding from the European Union's Horizon 2020 research and innovation programme under grant agreement No 762013 - NRG5 project.

\section{REFERENCES}

[1] Sexauer, J. Javanbakht, P., Mohagheghi, S., Phasor measurement units for the distribution grid: Necessity and benefits, 2013 IEEE PES Innovative Smart Grid Technologies Conference (ISGT), Washington, DC, USA

[2] Wang, D., Wilson, D., Venkata, S., Murphy, G., PMU-based angle constraint active management on $33 \mathrm{kV}$ distribution network, 2nd International Conference and Exhibition on Electricity Distribution (CIRED 2013), Stockholm, Sweden

[3] Zhang, C., Liu, Z., Huang, J., Wang, B., Wang, X., Fault location in active distribution network based on the change of current phase, 2017 IEEE Conference on Energy Internet and Energy System Integration (EI2), Beijing, China

[4] Ren, J., Venkata, S., Sortomme, E., An Accurate Synchrophasor Based Fault Location Method for Emerging Distribution Systems, IEEE Transactions on Power Delivery ( Volume: 29 , Issue: 1, Feb. 2014 )

[5] Macii, D., Barchi, G., Moser, D., Impact of PMUs on state estimation accuracy in active distribution grids with large PV penetration, 2015 IEEE Workshop on Environmental, Energy, and Structural Monitoring Systems (EESMS) Proceedings, Trento, Italy

[6] Amanda Kahunzire, Awodele, K., Improving distribution network state estimation by means of Phasor Measurement Units, 2014 49th International Universities Power Engineering Conference (UPEC), Cluj-Napoca, Romania

[7] Constandache, N., Stanescu, D., Sanduleac, M., Stanescu Carmen, Tristiu, I., Mandis, A., Smart Meters, PMU and PQ data analysis in Active Distribution Grids - Case Studies in MV networks, 2018 International Conference on Applied and Theoretical Electricity (ICATE), Craiova, Romania

[8] Nicorescu, H., Mihaela Albu, Eremia, M., Achieving interoperability of power system protection using SCADA and PMU information, 2019 8th International Conference on Modern Power Systems (MPS), Cluj Napoca, Romania

[9] Almas, M., Vanfretti, L., Løvlund, S., Gjerde, J., Open source SCADA implementation and PMU integration for power system monitoring and control applications, 2014 IEEE PES General Meeting | Conference \& Exposition, National Harbor, MD, USA

[10] Calin, M., Ana Maria Dumitrescu, Asprou, M., Kyriakides, E., Mihaela Albu, Measurement data aggregation for active distribution networks, 2013 IEEE International Workshop on Applied Measurements for Power Systems (AMPS), Aachen, Germany

[11] Su, H., Li, P., Fu, X., Yu, L., Chengshan Wang, Augmented Sensitivity Estimation Based Voltage Control Strategy of Active Distribution Networks With PMU Measurement, IEEE Access (Volume: 7), March 2019

[12] Popa. M., Calin, M., Dumitrescu, Ana Maria, Ciornei, S., Flexible and mobile deployment of synchronized measurement systems for active distribution grids, 2014 IEEE International Workshop on Applied Measurements for Power Systems Proceedings (AMPS), Aachen, Germany

[13] Project SUCCESS, https://success-energy.eu/, accessed 01.06.2019

[14] Project NRG5 - Energy as a Service, http://www.nrg5.eu/, accessed 01.06.2019

[15] Sanduleac, M., Lipari, G., Monti, A., et al, Next Generation Real-Time Smart Meters for ICT Based Assessment of Grid Data Inconsistencies, Energies 2017, 10(7), 857

[16] OpenDSS web site: http://smartgrid.epri.com/SimulationTool.aspx.

[17] OpenDSS documentation: https://sourceforge.net/projects/electricdss/ files /OpenDSS/

[18] GridMonk repository: https://github.com/MihSand/GridMonK 